Pontifícia U Uiversidade $C_{\text {atónlica }}$

Luiz Carlos da Silva Nunes

Análise da Técnica de Demodulação baseada em Filtros Fixos na interrogação de Sensores a Rede de Bragg em Fibras Ópticas

Tese de Doutorado

Tese apresentada como requisito parcial para obtenção do título de Doutor pelo Programa de PósGraduação em Engenharia Mecânica da PUC-Rio.

Orientadores: Arthur Martins Barbosa Braga Luiz Carlos Guedes Valente 


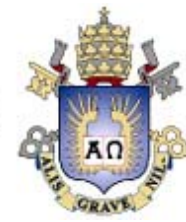

Luiz Carlos da Silva Nunes

\section{Análise da Técnica de Demodulação Baseada em Filtros Fixos para Interrogação de Sensores a Rede de Bragg em Fibras Ópticas}

Tese apresentada como requisito parcial para obtenção do grau de Doutor pelo Programa de Pós-Graduação em Engenharia Mecânica do Departamento de Engenharia Mecânica do Centro Técnico Científico da PUC-Rio. Aprovada pela Comissão Examinadora abaixo assinada.

Arthur Martins Barbosa Braga Orientador Pontifícia Universidade Católica do Rio de Janeiro

Luiz Carlos Guedes Valente Co-Orientador Gavea Sensors

Luis Carlos Blanco Linares Pontifícia Universidade Católica do Rio de Janeiro

Isabel Cristina dos Santos Carvalho Pontifícia Universidade Católica do Rio de Janeiro

Hypolito Jose Kalinowski CEFET/PR

Paulo Acioly Marques dos Santos Universidade Federal Fluminense

Adriana Lucia Cerri Triques Pontifícia Universidade Católica do Rio de Janeiro José Eugenio Leal Coordenador Setorial do Centro Técnico Científico - PUC-Rio 
Todos os direitos reservados. É proibida a reprodução total ou parcial do trabalho sem autorização da universidade, do autor e do orientador.

\section{Luiz Carlos da Silva Nunes}

Graduou-se Bacharel em Física na UFF (Universidade Federal Fluminense) em 1997. Recebeu o título de Mestre em Engenharia Mecânica na UFF em 2000. Tem como áreas de interesse a mecânica dos sólidos e a óptica aplicada. Participou de alguns congressos e publicou alguns artigos em revistas internacionais nas áreas de interesse.

Ficha Catalográfica

Nunes, Luis Carlos da Silva

Análise da técnica de demodulação baseada em filtros fixos na interrogação de sensores a rede de Bragg em fibra ópticas / Luiz Carlos da Silva Nunes ; orientadores: Arthur Martins Barbosa Braga, Luiz Carlos Guedes Valente .Rio de Janeiro : PUC-Rio, Departamento de Engenharia Mecânica, 2004.

120 f. : il. ; $30 \mathrm{~cm}$

Tese (doutorado) - Pontifícia Universidade Católica do Rio de Janeiro, Departamento de Engenharia Mecânica.

Inclui referências bibliográficas

1. Engenharia mecânica - Teses. 2. Rede de Bragg em fibras ópticas. 3. Sensores ópticos. 4. Técnica de demodulação. 5. Medida de temperatura. 6. Deformação e pressão. 7. Multiplexação (TDM/WDM). I. Braga, Arthur Martins Barbosa. II. Valente, Luiz Carlos Guedes. III. Pontifícia Universidade Católica do Rio de Janeiro. Departamento de Engenharia Mecânica. IV. Título. 


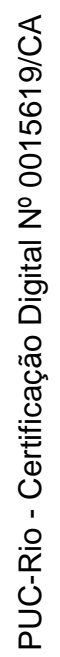

À minha família 


\section{Agradecimentos}

Aos meus orientadores Luiz Carlos Guedes Valente e Arthur Martins Barbosa Braga pelo apoio, conhecimento transferido, incentivo e amizade.

A Wolfgang Ecke e Reinhardt Willsch pelo apoio e conhecimento transferido.

Ao pessoal do Laboratório de Sensores a Fibra Óptica pelo apoio e amizade.

Ao pessoal do instituto IPHT pelo apoio e amizade.

A todos funcionários e professores que me ajudaram.

Ao grupo CS pelas conquistas.

Aos meus amigos que me ajudaram direta ou indiretamente.

A Capes pelo auxílio financeiro através da bolsa de estudos.

Ao DAAD pelo auxílio financeiro.

A Gavea Sensores.

A Deus por tudo. 


\section{Resumo}

Nunes, Luiz Carlos da Silva. Análise da Técnica de Demodulação baseada em Filtros Fixos na interrogação de Sensores a Rede de Bragg em Fibras Ópticas. Rio de Janeiro, 2004. 120p. Tese de Doutorado - Departamento de Engenharia Mecânica, Pontifícia Universidade Católica do Rio de Janeiro.

A análise da técnica de demodulação usada para interrogar sensores a rede de Bragg em fibras ópticas baseadas em filtros fixos foi realizada teoricamente e experimentalmente. Diferentes configurações de sistemas foram analisadas modificando a posição espectral dos filtros, assim como os níveis de potência óptica obtidos nos fotodetectores. Foram realizadas medidas com o tempo de integração que variavam de $0.01 \mathrm{a} 1 \mathrm{~s}$ e estimado o limite de baixa freqüência. Comparação entre os resultados experimentais e simulados mostram boa concordância, e extrapolações indicam que seria possível chegar a uma faixa de medida da ordem de 7 nanômetros, com incertezas equivalentes menores que 2 picometros, na medida da posição de pico do sensor. Foi feita uma análise da possibilidade de utilização desta técnica para medida simultânea de pressão e temperatura com uma única rede sensora. Na realização experimental foi usado um transdutor de pressão que transferia uma força transversal à fibra, proporcional à pressão atuante, gerando birrefringência na região da rede de Bragg. Foi possível obter valores de pressão com a faixa dinâmica de 400 psi com incerteza máxima de 4 psi e simultaneamente temperaturas com variação de 28 a $50^{\circ} \mathrm{C}$ com incerteza máxima de $0.1^{\circ} \mathrm{C}$. Adicionalmente, foram estudados os efeitos gerados em uma multiplexação temporal (TDM) de sensores a rede de Bragg quando os sensores se encontram superpostos na mesma posição espectral. Nesta análise é confrontada a técnica de demodulação utilizando dois filtros fixos com a técnica baseada na posição espectral. Os resultados indicam que a técnica baseada em filtros fixos apresenta vantagem, permitindo um número significativamente maior de sensores. E também, foi analisado o distúrbio provocado no espectro da rede sensora quando a fonte de luz usada para interrogar a rede tem uma modulação espectral que varia com a temperatura. Finalmente, foi apresentado um sistema de multiplexação (TDM/WDM), completamente polarizado, capaz de interrogar dezenas de sensores a rede de Bragg escrito em fibras de alta birrefringência. $\mathrm{O}$ sistema de multiplexação consistiu em uma chave óptica integrada, baseada em um interferômetro de Mach-Zehnder com configuração X-Y, e em um espectrômetro com um CCD linear como elemento de detecção.

\section{Palavras-chave}

Rede de Bragg em fibras ópticas; sensores ópticos; técnica de demodulação; medida de temperatura, deformação e pressão; multiplexação (TDM/WDM) 


\section{Abstract}

Nunes, Luiz Carlos da Silva. Analysis of the Demodulation Technique based on fixed filters in the interrogation of Fiber Bragg Grating Sensors. Rio de Janeiro, 2004. 120p. Tese de Doutorado - Departamento de Engenharia Mecânica, Pontifícia Universidade Católica do Rio de Janeiro.

The analysis of a demodulation system for fiber Bragg grating sensors based on two fixed spectral filters has been carried out both theoretically and experimentally. Different system configurations were analyzed by modifying the spectral position of the filters as well as the optical power-level of the signal reaching the two photo-detectors. Measurements with integration times that varied from 0.01 to 1 second have been compared with the low frequency limit predicted for long-term operation. Comparisons between simulated and experimental results show good agreement, and extrapolations indicate that it should be possible to achieve a dynamic range of the order of 7 nanometers, with uncertainties equivalent to less than 2 picometers, in measurements of the sensor peak position. Applications based on this system were carried out. An analysis of simultaneous measurement of temperature and pressure with only one FBG sensor using transducer of pressure to transfer a lateral force to the fiber, proportional to the applied pressure, generating birefringence at grating Bragg region has been realized. The proposed system allowed to measure pressure range of 400 psi with uncertain of 4 psi and simultaneously temperature range of $22^{\circ} \mathrm{C}$ with uncertain of $0.1{ }^{\circ} \mathrm{C}$. In addiction, generated effects in the temporal multiplexing (TDM) of fiber Bragg grating sensors when the sensor spectra are fully overlapped have been analyzed. In this study, it is compared the demodulation technique based on two fixed filters with the conventional technique based on the peak position. The results show that the technique based on fixed filters presents advantage to conventional, allowing a greatest sensor number. It was also analyzed the generated disturbance in the Bragg grating sensor spectrum when the source used to interrogate the sensor has a residual modulation which changes with the temperature. And finally, a polarized multiplexing system (TDM/WDM) able to interrogate a large number of Bragg grating sensors written in high-birefringent polarization-maintaining fibers has been realized. It is based on integrated-optic switch Mach-Zehnder interferometer in X-Y configuration and a CCD line array spectrometer.

\section{Keywords}

Bragg grating in optical fiber; optical sensors; demodulation technique; measurement of temperature, deformation and pressure; multipleting (TDM/WDM). 


\section{Sumário}

1 Introdução

2 Conceitos preliminares $\quad 21$

2.1. Introdução $\quad 21$

2.2. Propriedades da rede de Bragg em fibras ópticas 21

2.2.1. Rede de Bragg 21

2.2.2. Refletividade da rede de Bragg uniforme 24

2.2.3. Sensibilidade da rede de Bragg em função da temperatura e da deformação26

2.2.4. Análise da rede de Bragg sujeita a compressão transversal 27

2.3. Técnicas de leitura 31

2.3.1. Circuito óptico utilizando Analisador de Espectro Óptico (OSA-Optical Spectrum Analyzer) 31

2.3.2. Técnicas de Demodulação 32

2.3.3. Demodulação óptica utilizando um filtro fixo 34

2.3.4. Demodulação óptica utilizando dois filtros fixos 36

3 Análise do sistema baseado em demodulação óptica usando dois filtros fixos para interrogar sensores a rede de Bragg em fibras ópticas 38

3.1. Introdução 38

3.2. Modelo teórico 39

3.3. Montagem experimental e resultados 44

3.4. Sistema com a largura de banda à meia altura aumentada 51

3.5. Validação teórico-experimental 53

4 Medida simultânea de temperatura-pressão com uma única rede de Bragg usando demodulação baseada em dois filtros fixos 56

4.1. Introdução 56

4.2. Sensor a rede de Bragg submetido a uma compressão transversal 56

4.3. Montagem experimental e resultados 57

4.4. Simulação numérica e validação teórico-experimental 64 
5 Análise do desvio provocado no comprimento de onda de Bragg do sensor devido a mudanças no espectro da luz incidente

5.1. Introdução

5.2. Análise do desvio no comprimento de onda de Bragg gerado na multiplexação (TDM/WDM) de sensores 72

5.2.1. Multiplexação de sensores: modelo teórico e simulação 73

5.2.2. Montagem experimental e resultados 82

5.3. Análise do desvio no comprimento de onda de Bragg devido à variação da modulação residual no espectro da fonte de luz

5.3.1. Modelo teórico

5.3.2. Resultados e discussão

6 Multiplexação de sensores a rede de Bragg em fibra ópticas de alta birrefringência baseada em TDM/WDM 99

6.1. Introdução 99

6.2. Sistema de Multiplexação TDM/WDM 100

$\begin{array}{ll}\text { 6.2.1. Chave óptica integrada } & 101\end{array}$

6.2.2. Espectrômetro com um CCD linear como elemento de detecção 101

6.2.3. Conjunto de sensores a rede de Bragg 102

6.3. Problemas com deriva nos níveis de voltagens aplicadas à chave óptica e sua correção

6.4. Resultados

6.5. Aplicação do sistema de multiplexação na medida de compressão transversal

7 Conclusão

Referências bibliográficas

Apêndice A

Teoria da convolução 


\section{Lista de figuras}

Figura 2.1 - Esquema representativo para a dedução da equação de Bragg. $\quad 22$

Figura 2.2 - Reflexão e transmissão da rede de Bragg 23

Figura 2.3 - Espectro da refletividade de uma rede de Bragg homogênea como uma função do comprimento de onda 25

Figura 2.4 - Espectro refletido de uma rede de Bragg homogênea sujeita a compressão transversal

Figura 2.5 - Visão esquemática da rede de Bragg sujeita a uma força transversal:

(a) corte longitudinal; (b) corte transversal $A A$

Figura 2.6 - Variação do comprimento de onda versus a força aplicada: (a) caso "plane strain" $\left(\varepsilon_{\mathrm{z}}=0\right)$ e (b) "plane stress" $\left(\sigma_{\mathrm{z}}=0\right)$

Figura 2.7 - Circuito óptico utilizando Analisador de Espectro Óptico 32

Figura 2.8 - (a) Princípio do método do filtro passa banda; (b) método de filtro ajustável; (c) método de varredura interferométrica 34

Figura 2.9 - Sistema óptico utilizando um filtro fixo 34

Figura 2.10 - Interseção entre os espectros sensor-filtro 35

Figura 2.11 - Leitura relativa ao fotodetector DET 1

Figura 2.12 - Circuito óptico proposto nesta tese, utilizando dois filtros fixos 36

Figura 3.1 - Espectro de transmissão dos filtros (pico em $1540.1 \mathrm{~nm}$ e $1542.2 \mathrm{~nm}$ ) e espectro refletido do sensor (pico em 1541.3) 40

Figura 3.2 - Resultados numéricos e experimentais relativos aos dois fotodetectores, $V_{l}$ e $V_{2}$, em função do comprimento de onda do pico espectral do sensor para uma separação entre os filtros de $5 \mathrm{~nm}$

Figura 3.3 - Resultados numéricos e experimentais para a função $f$ em função do comprimento de onda do pico espectral do sensor obtido a partir dos dados da figura 3.2

Figura 3.4 - Incerteza teórica e experimental no limite de baixa freqüência para uma separação entre filtros de $5 \mathrm{~nm}$

Figura 3.5 - Montagem experimental

Figura 3.6 - Voltagem obtida em função da posição de pico do sensor para separações espectrais entre filtros (SF) de 4, 5 e 6 nm. Para separação 
entre filtros de $5 \mathrm{~nm}$, foram usados dois níveis de potência

Figura 3.7 - Função $f=V_{1} / V_{2}$ normalizada correspondente às mesmas situações apresentadas na figura 3.6. Para o sistema com um "único filtro" foram considerados os dados de máxima potência, figura 3.6

Figura 3.8 - Incertezas para o tempo de integração de 1 segundo e para $0.1 \%$ no limite de baixa freqüência para diferentes separações entre filtros: 4 $\mathrm{nm}(\mathrm{a}), 5 \mathrm{~nm}$ (b), $6 \mathrm{~nm}$ (c) e único filtro (d)

Figura 3.9 - Incerteza para tempo de integração de 1 segundo, para uma separação de filtros de $5 \mathrm{~nm}$ com dois níveis de potência que diferem por um fator 6. Os dados obtidos com maior potência foram multiplicados por 6

Figura 3.10 - (a) Incerteza no comprimento de onda para tempos de integração de 0.01, 0.1 e $1 \mathrm{~s} \mathrm{com} \mathrm{separação} \mathrm{entre} \mathrm{filtros} \mathrm{de} 4 \mathrm{~nm}$, e (b) dependência da raiz quadrada da relação sinal ruído com respeito à largura de banda do fotodetector

Figura 3.11 - Simulação para um sistema com largura de banda a meia altura de 4 $\mathrm{nm}$ para os filtros e $1.5 \mathrm{~nm}$ para o sensor e com uma separação entre filtros de $11 \mathrm{~nm}$, e também o resultado para um único filtro: (a) Saída nos dois fotodetectores $V 1$ e $V 2$; (b) Função $f=(V 1 / V 2)$ e $f=V 1$, (c) Incerteza no limite de baixa freqüência

Figura 3.12 - (a) Espectros do sensor, dos filtros e da interseção entre eles, (b) área de interseção entre os espectros FS

Figura 3.13 - (a) Curva de convolução associada às potências lidas nos

fotodetectores; (b) razão entre as convoluções, $f$. Curva sólida:

simulação; pontos: dados experimentais

Figura 4.1 - Esquema experimental do sistema capaz de realizar medidas simultâneas de temperatura e pressão, usando filtros fixos

Figura 4.2 - Espectros dos filtros e do sensor (FBG) sujeito a uma pré-carga 59

Figura 4.3 - Variação do comprimento de onda do sensor em função da pressão para diferentes temperaturas

Figura 4.4 - Potência óptica lida nos fotodetectores 1 e 2

Figura 4.5 - (a) potência óptica lida no fotodetector 1; (b) potência óptica lida no fotodetector 2; (c) linhas de mesma potência, fotodetectores 1 e $2 \quad 62$ 
Figura 4.6 - Incerteza estimada para medida da pressão 64

Figura 4.7 - Incerteza estimada para medida da temperatura 64

Figura 4.8 - (a) Espectro do sensor a rede de Bragg com e sem pré-carga. (b)

Espectro dos filtros. Pontos: resultados experimentais; linhas sólidas: simulação

Figura 4.9 - Ajuste da variação do comprimento de onda de Bragg em função da temperatura: (a) primeiro pico, (b) segundo pico

Figura 4.10 - Ajuste da variação do comprimento de onda em função da pressão:

(a) primeiro pico, (b) segundo pico

Figura 4.11 - Distribuição dos valores de potência normalizada no fotodetector 1 em função da temperatura e da pressão. (a) Experimental; (b) simulação

Figura 4.12 - Distribuição dos valores de potência normalizada no fotodetector 2 em função da temperatura e da pressão. (a) Experimental; (b) simulação

Figura 4.13 - Distribuição dos valores de potência normalizada, fotodetectores 1

(a) e 2 (b) 70

Figura 4.14 - Incerteza na pressão (a) e na temperatura (b) 70

Figura 5.1 - Multiplexação de sensores com espectros idênticos 74

Figura 5.2 - Sinal transmitido que incide no sensor $n$ em função do número de sensores

Figura 5.3 - Intensidade do espectro refletido do sensor $n$ em função do número de sensores

Figura 5.4 - Intensidade transmitida para três diferentes casos, considerando o número de sensores, o percentual de refletividade e a quantidade de sensores como variáveis

Figura 5.5 - (a) Espectro dos filtros DWDM e do sensor; (b) função razão $f$ em função do comprimento de onda de Bragg

Figura 5.6 - Desvio para diferentes percentuais de refletividade, com 100 sensores na mesma posição espectral, $1545 \mathrm{~nm}$

Figura 5.7 - Desvio para diferentes números de sensores na mesma posição espectral, $1545 \mathrm{~nm}$, com percentual de refletividade de $1 \%$

Figura 5.8 - Desvio calculado para valores aleatórios: comprimento de onda e 
refletividade variando de 1543.5 a $1543.7 \mathrm{~nm}$ e 0.5 a $1.0 \%$, respectivamente, com 100 sensores $\quad 80$

Figura 5.9 - Máximo desvio para diferentes posições do distúrbio, filtros fixos 81 Figura 5.10 - Esquema experimental usado para representar um conjunto de sensores idênticos na mesma posição espectral $\quad 82$ Figura 5.11 - Espectros da rede de Bragg fixa e do filtro de transmissão tipo Fabry-Perot, variável. Resultados experimentais e simulados 83

Figura 5.12 - Espectros do filtro ajustável, da fonte após passar pela rede de Bragg e do resultado com distúrbio

Figura 5.13 - Espectro observado do filtro tipo Fabry-perot após passar pela rede de Bragg fixa em algumas posições distintas: (a) dados experimentais, (b) simulação

Figura 5.14 - Comparação entre o comprimento de onda do filtro ajustável indicado no analisador de espectro óptico e o seu valor verdadeiro para os casos onde o espectro da luz incidente sofre ou não a influência da rede fixa

Figura 5.15 - Desvio provocado na posição espectral devido ao distúrbio gerado $\begin{array}{ll}\text { pela rede fixa } & 87\end{array}$

Figura 5.16 - Espectros do DWDM, usados como filtros 88

Figura 5.17 - (a) Função gerada pela razão entre as potências obtidas no medidor de potência óptica versus o comprimento de onda do filtro ajustável para os casos com e sem influência da rede fixa; (b) visualização em escala ampliada, região do circulo.

Figura 5.18 - Comparação entre o desvio obtido na leitura da posição de pico espectral e no uso da técnica de filtros fixos

Figura 5.19 - Análise do método baseado em posição de pico. (a) Espectros da fonte com modulação, do sensor e da interseção entre eles; (b) espectro da interseção fonte-sensor e o ajuste polinomial; (c) Desvio na posição de pico com e sem ajuste.

Figura 5.20 - Análise baseada no método de dois filtros fixos: (a) Espectros dos filtros e da interseção fonte-sensor; (b) Convoluções e razão entre elas; (c) Variação da função razão

Figura 5.21 - Desvio na posição de pico considerando o método baseado na 
posição espectral, para dois diferentes casos: variação do FWHM do sensor e da amplitude da modulação da fonte 95

Figura 5.22 - Método de dois filtros fixos. (a) função razão; (b) variação da função razão. Para dois diferentes casos: variação do FWHM do sensor e da amplitude da modulação da fonte 96

Figura 5.23 - (a) função razão $f$ e máxima variação da função razão ; (b) Máximo desvio da posição. Para diferentes posições do sensor 98

Figura 6.1 - Esquema do sistema de multiplexação TDM/WDM 100

Figura 6.2 - (a) Chave óptica integrada, (b) Perfil da voltagem aplicada na chave óptica

Figura 6.3 - Espectrômetro utilizado para realização da multiplexação do comprimento de onda

Figura 6.4 - (a) Espectros de um grupo de sensores obtidos com os níveis de voltagens aplicados na chave óptica: sendo ele sem deriva, completamente bloqueado; com deriva , não bloqueado e corrigido, subtração do sinal de erro; (b) Sensor A, com o espectro normalizado; (c) Sinal referente ao erro espectral gerado com os níveis de voltagem com e sem deriva.

Figura 6.5 - Espectros observados nos quatro intervalos de tempo: 120, 280, 480 e $720 \mathrm{~ns}$, relativos a região de referência (sem sensores), (a), e aos três grupos de sensores, (b), (c) e (d)

Figura 6.6 - (a) Espectro refletido de um grupo de sensores com os dois modos de polarização independentes. (b) espectro de um sensor obtido com o auxilio do OSA, usando luz não polarizada

Figura 6.7 - Rede de Bragg em fibra óptica submetida à compressão transversal109 Figura 6.8 - (a) Intensidade e (b) comprimento de onda de Bragg versus diferente ângulos de aplicação de força. Força aplicada antes da rede de Bragg

Figura 6.9 - Variação do comprimento de onda de Bragg, em função da força aplicada

Figura A.1 - (a) Interseção dos pulsos para diferentes valores de $u$; (b) 


\section{Lista de tabelas}

Tabela 1.1 - Sensores ópticos para várias aplicações

Tabela 3.1 - Valores experimentais da faixa de operação em nm onde a incerteza da posição de pico do comprimento de onda está abaixo de 1 e 10 pm. 
Perguntei-lhe onde mandara fazê-lo, e ele respondeu que ele mesmo o fizera, e quando perguntei onde conseguira suas ferramentas, disse que ele mesmo as tinha feito e, rindo, acrescentou: "Se eu fosse esperar que outras pessoas fizessem minhas ferramentas e tudo o mais para mim, eu nunca teria feito nada".

(Lembrança de Newton, um ano antes de morrer, sobre a construção do primeiro telescópio refletor; recordada por John Conduitt, casado com a sobrinha do cientista) 